\title{
PREVENÇÃO E TRATAMENTO DE RADIODERMATITE: UMA REVISÃO INTEGRATIVA*
}

Franciane Schneider ${ }^{1}$, Edivane Pedrolo 2 , Jolline Lind ${ }^{3}$, Alessandra Amaral Schwanke Mitzy Tannia Reichembach Danski ${ }^{5}$

\begin{abstract}
RESUMO: Objetivou-se levantar tecnologias para prevenção e/ou tratamento de radiodermatites em pacientes com câncer de cabeça e pescoço. Desenvolveu-se uma revisão integrativa da literatura no mês de julho de 2011 nas bases de dados Biblioteca Nacional de Medicina dos Estados Unidos e Literatura Latino-Americana e do Caribe em Ciências da Saúde, utilizando-se a estratégia PICO (população, intervenção, comparação, desfecho ou out comes) para a busca de artigos que respondessem a questão norteadora, obtendo-se cinco artigos. Os resultados dos ensaios clínicos não trouxeram diferenças significativas na utilização dos produtos ou cuidados na prevenção e/ou tratamento de radiodermatite em câncer de cabeça e pescoço; o único estudo que conclui a eficácia de um produto desenvolveu-se sem comparações. Infere-se não haver evidência científica para a introdução de um produto/cuidado para prevenção e/ou tratamento de radiodermatite a partir dos resultados deste estudo.

DESCRITORES: Radiodermatite; Enfermagem; Radioterapia; Tecnologia; Difusão de inovações.
\end{abstract}

\section{PREVENTION AND TREATMENT OF RADIODERMATITIS: AN INTEGRATIVE REVIEW}

ABSTRACT: The aim was to survey technologies for the prevention and/or treatment of radiodermatitis in patients with cancer of the head and neck. An integrative review was undertaken of the literature in the month of July 2011 in the following databases: the National Library of Medicine and Latin American and Caribbean Health Sciences, using the PICO strategy (Population, Intervention, Comparison, and Outcomes) for searching for articles which respond to the guiding question. Five articles were obtained. The results of the clinical trials did not raise significant differences in the use of the products or cares in the prevention and/or treatment of radiodermatitis in cancer of head and neck; the only study which concluded a product to be efficacious was carried out without comparisons. It is inferred that there is no scientific evidence for the introduction of a product/care measure for the prevention and/or treatment of radiodermatitis based on the results of this study.

DESCRIPTORS: Radiodermatitis; Nursing; Radiotherapy; Technology; Diffusion of innovations.

\section{PREVENCIÓN Y TRATAMIENTO DE RADIODERMATITIS: UNA REVISIÓN INTEGRATIVA}

RESUMEN: El objetivo de este estudio fue verificar tecnologías para prevención y/o tratamiento de radiodermatitis en pacientes con cáncer de cabeza y cuello. Se desarrolló una revisión integrativa de la literatura en el mes de julio de 2011 en las bases de datos Biblioteca Nacional de Medicina de Estados Unidos y Literatura Latinoamericana y del Caribe en Ciencias de la Salud, utilizándose la estrategia PICO (población, intervención, comparación, desenlace o out comes) para la búsqueda de artículos que contestasen a la cuestión base, obteniéndose cinco artículos. Los resultados de los ensayos clínicos no presentaron diferencias significativas en la utilización de los productos o cuidados en la prevención y/o tratamiento de radiodermatitis en cáncer de cabeza y cuello; el único estudio que concluye la eficacia de un producto se desarrolló sin comparaciones. Se deduce que no hay evidencia científica para la introducción de un producto/cuidado para prevención y/o tratamiento de radiodermatitis a partir de los resultados de este estudio. DESCRIPTORES: Radiodermatitis; Enfermería; Radioterapia; Tecnología; Difusión de innovaciones.

*Artigo relacionado à dissertação de mestrado do Programa de Pós-graduação em Enfermagem da Universidade Federal do Paraná PPGENF UFPR, intitulada "Avaliação da eficácia da Calendula officinalis na prevenção e tratamento de radiodermatite de cabeça e pescoço".

'Enfermeira do Centro de Oncologia do Hospital Santa Catarina de Blumenau. Especialista em Oncologia. Mestranda pelo PPGENF UFPR. Bolsista CAPES. Membro do Grupo de Pesquisa Tecnologia e Inovação em Saúde: Fundamentos para a prática profissional- TIS. ${ }^{2}$ Enfermeira. Mestranda pelo PPGENF UFPR. Professora do Instituto Federal do Paraná. Membro do TIS.

${ }^{3}$ Acadêmica em Enfermagem da UFPR. Bolsista CNPq de Iniciação Científica. Membro do TIS.

${ }^{4}$ Acadêmica em Enfermagem da UFPR. Bolsista voluntária de Iniciação Científica. Membro do TIS.

${ }^{5}$ Enfermeira. Doutora. Professora da Departamento de Enfermagem e do PPGENF UFPR. Vice-líder do TIS.

Autor correspondente:

Recebido: $29 / 08 / 2012$

Franciane Schneider.

Aprovado: 19/12/2012

Universidade Federal do Paraná

Av. Sete de Setembro, 1045 - 80050-255 - Curitiba-PR-Brasil

E-mail: franciane_06@yahoo.com.br 


\section{INTRODUÇÃO}

O câncer de cabeça e pescoço é um termo coletivo utilizado para descrever tumores malignos das seguintes regiões anatômicas: lábio e cavidade oral, faringe, laringe, seios maxilares, cavidade nasal, seios paranasais/ etmoidais, glândulas salivares e tireóide ${ }^{(1)}$. Os principais fatores de risco são tabagismo, etilismo, condições de higiene bucal precárias, dentre outros ${ }^{(2)}$. Destaca-se que o hábito de fumar e beber, de forma concomitante, estabelece um sinergismo que aumenta em até 30 vezes o risco para o desenvolvimento desse tipo de câncer ${ }^{(3)}$.

A radioterapia é a principal modalidade de tratamento para combater o câncer de cabeça e pescoço( ${ }^{(4)}$ eé realizada de forma loco-regional, objetivando a cura, remissão, profilaxia ou paliação ${ }^{(5)}$. É definida como o uso terapêutico de radiações ionizantes, tendo como finalidade destruir e/ou inibir o crescimento de células tumorais do organismo, impedindo sua multiplicaçãopor mitose e/ou determinando a sua morte celular ${ }^{(5-7)}$.

Um dos efeitos adversos mais comuns do tratamento radioterápico é a radiodermatite, também conhecida como radiodermite. É definida como um conjunto de lesões cutâneas provocadas por uma exposição excessiva à radiação ionizante, a qual leva à desidratação da pele e pode ocasionar complicações graves, como ulceração, ou complicações secundárias, como infecção local. É uma reação cutânea que está limitada ao campo de tratamento de radiação ou ao seu ponto de saída ${ }^{(8-10)}$. Considera-se que $95 \%$ dos pacientes tratados com radioterapia desenvolvem alguma forma de reação de pele ${ }^{(11)}$.

Destaca-se que a radiodermatite é um efeito adverso que pode ser prevenido, ou minimizado, por meio de orientações aos pacientes, aos familiares e/ou acompanhantes/cuidadores sobre os cuidados com a pele, uma vez que esses o realizam também em domicílio; e de intervenções precoces, contribuindo com a integridade cutânea do local irradiado. Os enfermeiros interagem de forma direta com os pacientes e cabe a esses profissionais oferecer as informações necessárias para que o cuidado seja mantido, com segurança e qualidade.

Destarte, objetivou-se levantar tecnologias para prevenção e/ou tratamento de radiodermatites em pacientes com câncer de cabeça e pescoço.

\section{MÉTODO}

Desenvolveu-se uma revisão integrativa da literatura, a qual permite a formulação de conclusões gerais a respeito de determinada área de estudo, mediante síntese de múltiplos estudos publicados ${ }^{(12)}$.
A pesquisa foi realizada no mês de julho de 2011 nas bases de dados Biblioteca Nacional de Medicina dos Estados Unidos (PubMed) e Literatura Latino -Americana e do Caribe em Ciências da Saúde (LILACS). Para essa busca, empregaram-se os descritores de assunto do Descritor em Ciências da Saúde (DeCS) pela interface BIREME, e do Medical Subject Heading (MeSH) pelo PubMed.

A questão norteadora da presente revisão foi: Qual a terapêutica mais eficaz para prevenção e/ou tratamento de radiodermatites em pacientes com neoplasias de cabeça e pescoço em tratamento radioterápico: calêndula ou ácidos graxos essenciais? Utilizou-se a estratégia PICO para busca dos $\operatorname{artigos}^{(13)}$, a qual viabiliza o processo de encontrar respostas apropriadas às dúvidas advindas da prática ${ }^{(14)}$. A estratégia empregada encontra-se descrita no quadro 1.

Utilizaram-se três estratégias de busca: 1. Cruzamento por descritores: Neoplasias de cabeça e pescoço $A N D$ Medicamentos fitoterápicos $O R$ Calêndula $A N D$ Ácidos graxos essenciais $A N D$ Radiodermatite; 2 . Cruzamentos por palavras: Neoplasias de cabeça e pescoço $A N D$ Medicamentos fitoterápicos $A N D$ Radiodermatite; Neoplasias de cabeça e pescoço $A N D$ Medicamentos fitoterápicos $A N D$ Ácidos graxos essenciais $A N D$ Radiodermatite; Neoplasias de cabeça e pescoço $A N D$ Medicamentos fitoterápicos $A N D$ Ácidos graxos essenciais; Neoplasias de cabeça e pescoço $A N D$ Medicamentos fitoterápicos $A N D$ Calêndula $A N D$ Ácidos graxos essenciais $A N D$ Radiodermatite; Neoplasias de cabeça e pescoço $A N D$ Medicamentos fitoterápicos $A N D$ Calêndula $A N D$ Ácidos graxos essenciais; Calêndula $A N D$ Radiodermatite; Ácidos graxos essenciais AND Radiodermatite; e 3. Revisão da bibliografia dos artigos selecionados.

Posteriormente à busca, procedeu-se a leitura dos títulos e resumos dos artigos identificados, dentre os quais, foram selecionados aqueles que atenderam aos critérios de inclusão: ser publicado entre o período de janeiro de 2001 e junho de 2011; em periódicos nacionais e internacionais; estar disponíveis nos idiomas português, inglês ou espanhol; abordar temas relacionados à prevenção e/ou tratamento de radiodermatite em pacientes com câncer de cabeça e pescoço na população adulta.

A busca na base de dados LILACS totalizou 134 referências, das quais somente uma atendeu aos critérios de inclusão. Na PubMed obteve-se 126 artigos, dois quais quatro foram incluídos na presente revisão. Desta forma, a amostra final correspondeu a cinco artigos.

Para extração dos dados empregou-se instrumento de coleta previamente testado, contendo: identificação do artigo; titulação dos autores, tema, objetivo, metodologia empregada, nível de evidência, amostra/população do estudo, local/país do estudo, constituição dos grupos inter- 
venção e controle, resultados e conclusões. O nível de evidência foi avaliado de acordo com a classificação proposta pela Oxford Center for Evidence Based Medicine, que compreende 10 níveis de evidência ${ }^{(15)}$. As etapas de discussão e interpretação dos resultados, bem como síntese dos dados extraídos são apresentados de forma descritiva.

Quadro 1 - Descritores de assunto empregados na busca dos artigos. Curitiba, 2011

\begin{tabular}{|l|l|}
\hline Grupo & Descritores de assunto \\
\hline População ou paciente & NEOPLASIAS DE CABEÇA E PESCOÇO \\
\hline Intervenção ou indicador & MEDICAMENTOS FITOTERÁPICOS or CALÊNDULA \\
\hline Controle ou comparação & ÁCIDOS GRAXOS ESSENCIAIS \\
\hline Outcome ou desfecho & RADIODERMATITE \\
\hline
\end{tabular}

\section{RESULTADOS}

Evidencia-se, em relação ao desenho do estudo, que dos cinco artigos inclusos, umé de revisão integrativa de literatura e quatro são ensaios clínicos, sendo três deles randomizados. Destaca-se no quadro 2 as características de cada estudo em relaçãoaos objetivos, desenho depesquisa enível de evidência.

Observa-se, conforme o quadro 3 , que os resultados e conclusões dos ensaios clínicos ${ }^{(16-19)}$ não trouxeram diferenças significativas na utilização dos produtos ou cuidados na prevenção e/ou tratamento de radiodermatite. A revisão integrativa constata que não há predominância de nenhum produto ou curativo para utilização na prática diária. O único estudo que conclui a eficácia de um produto, dismutase superoxidase, desenvolveu-se sem comparações (Evidência 2C).

Salienta-se, conforme o quadro 4, que em dois ensaios clínicos os participantes eram portadores de câncer de cabeça e pescoço, exclusivamente, e que nos demais foram incluídos participantes com câncer de cabeça e pescoço, mama, próstata, reto, dentre outras localizações. Nota-se que em dois dos ensaios clínicos obteve-se um número considerável e relevante de participantes (acima de 350), que em três as intervenções compararam a utilização de no mínimo dois produtos/cuidados na prevenção e/ou tratamento de radiodermatite e que dois não possuem grupo controle.

Quadro 2 - Características dos estudos em relação aos objetivos, desenho de pesquisa e nível de evidência. Curitiba, 2012

\begin{tabular}{|c|c|c|}
\hline Referência, ano & Objetivos & $\begin{array}{l}\text { Desenho de estudo } \\
\text { (Nível de Evidência) }\end{array}$ \\
\hline 5,2006 & $\begin{array}{l}\text { Levantar o estado da arte quanto ao tratamento de radioderma- } \\
\text { tite em pacientes oncológicos, analisando quantitativamente os } \\
\text { achados publicados na literatura científica quanto à utilização, } \\
\text { efetividade e frequência; identificar os produtos e curativos em- } \\
\text { pregados de maneira tópica por profissionais da saúde nestas } \\
\text { lesões; e contribuir para as intervenções de enfermagem no } \\
\text { cuidado e tratamento da radiodermatite ao paciente oncológico }\end{array}$ & $\begin{array}{l}\text { Revisão de literatura } \\
\text { sistematizada sem me- } \\
\text { tanálise, utilizando-se } \\
\text { as bases de dados Lilacs, } \\
\text { Medline, Pubmed e Ci- } \\
\text { nahl no período de } 1993 \\
\text { a } 2004 \text { (Não se aplica) } \\
\end{array}$ \\
\hline 16,2002 & $\begin{array}{l}\text { Avaliar possível diferença entre a utilização de solução em creme ou } \\
\text { em pó; avaliar se o paciente sentiu diferença entre uma concepção de } \\
\text { cuidado em comparação com a outra e se os médicos viram diferença } \\
\text { na extensão das reações cutâneas agudas nos campos de radiação; } \\
\text { avaliar se uma das concepções foi capaz de prevenir o aparecimento } \\
\text { ou reduzir a extensão da descamação úmida }\end{array}$ & $\begin{array}{l}\text { Ensaio clínico rando- } \\
\text { mizado, controlado, } \\
\text { não cego (Evidência } \\
\text { 2B) }\end{array}$ \\
\hline 17,2008 & $\begin{array}{l}\text { Avaliar a eficiência do dismutase superoxidase (SOD) administrado } \\
\text { topicamente no tratamento de radiodermatite aguda, bem como a sua } \\
\text { capacidade em evitar a interrupção de tratamentos de radioterapia }\end{array}$ & $\begin{array}{l}\text { Ensaio clínico aberto } \\
\text { (Evidência } 2 \mathrm{C} \text { ) }\end{array}$ \\
\hline 18,2006 & $\begin{array}{l}\text { Comparar o uso de emulsão de Trolamine }{ }^{\circledR} \text {, como agente profilático ou } \\
\text { como agente de intervenção, com produto padrão usado na instituição } \\
\text { para redução da incidência de radiodermatite de alto grau }\end{array}$ & $\begin{array}{l}\text { Ensaio clínico randomi- } \\
\text { zado, controlado, não } \\
\text { cego. (Evidência 1B) } \\
\end{array}$ \\
\hline 19, 2004 & $\begin{array}{l}\text { Examinar o efeito de creme aquoso, creme de sucralfato ou nenhum creme, } \\
\text { no desenvolvimento de reações de pele e desconfortos associados, decor- } \\
\text { rentes da radioterapia em câncer de cabeça e pescoço, mama ou anorretal }\end{array}$ & $\begin{array}{l}\text { Ensaio clínico rando- } \\
\text { mizado, controlado, } \\
\text { cego (Evidência } 1 \mathrm{~B})\end{array}$ \\
\hline
\end{tabular}


Quadro 3 - Síntese dos estudos em relação aos principais resultados e conclusões. Curitiba, 2012

\begin{tabular}{|c|c|}
\hline Referência, ano & Resultados e conclusões \\
\hline 5,2006 & $\begin{array}{l}\text { Levantados } 16 \text { periódicos nacionais e internacionais; principais regiões de investiga- } \\
\text { ção: mama (32\%) e cabeça e pescoço (32\%). Radiodermatite: não ocorreu predominân- } \\
\text { cia de algum produto ou curativo no tratamento dessa reação que pudesse ser sugerido } \\
\text { para aplicação na prática, bem como muitos não estão disponíveis no mercado nacional. } \\
\text { Os estudos abordam reações até quatro semanas pós-tratamento, e não reações tardias }\end{array}$ \\
\hline 16,2002 & $\begin{array}{l}\text { Avaliação subjetiva: ardência local, tensão, prurido, dor e desconforto - não houve diferença en- } \\
\text { tre os produtos (creme ou pó). Avaliação objetiva: não pode ser comprovado um efeito relevante } \\
\text { sobre o início de reações agudas ou diferenças de alívio dos sintomas entre os dois tipos de tra- } \\
\text { tamento; não houve diferença no grau ou o aparecimento de descamações úmidas. Os dois tipos } \\
\text { de tratamento foram considerados favoráveis aos cuidados com a pele exposta a radioterapia }\end{array}$ \\
\hline 17,2008 & $\begin{array}{l}\text { A dismutase superoxidase (SOD) administrada por via tópica é eficaz e pode controlar a } \\
\text { radiodermatite. A SOD ainda mostra } 77,1 \% \text { de resposta parcial ou total no final da radiotera- } \\
\text { pia. Não foi observado agravamento de toxicidade aguda no final do período de } 12 \text { semanas } \\
\text { e a remissão da toxicidade foi total. Conclui-se que a administração tópica de SOD é uma } \\
\text { alternativa eficaz para o tratamento de radiodermatite }\end{array}$ \\
\hline 18,2006 & 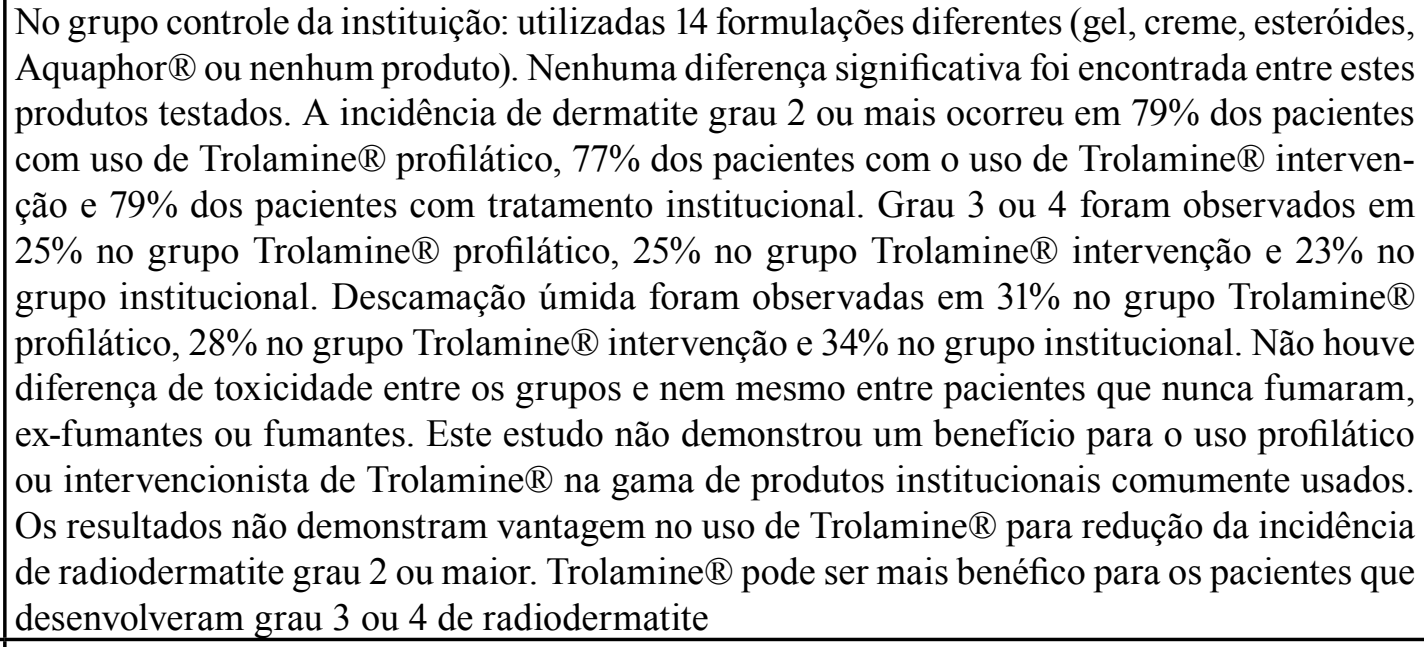 \\
\hline 19,2004 & $\begin{array}{l}\text { Não foram encontradas diferenças significativas na severidade da reação cutânea ou nível de } \\
\text { desconforto do paciente nos grupos analisados. Pacientes tratados concomitantemente com } \\
\text { quimioterapia obtiveram reações de pele significativamente piores. Pacientes fumantes ou } \\
\text { ex-fumantes estão mais predispostos a desenvolver radiodermatite. Houve também diferen- } \\
\text { ças entre as áreas de tratamento, com pacientes de cabeça e pescoço apresentando as piores } \\
\text { reações nas avaliações dos pesquisadores e dos próprios pacientes, assim como os escores } \\
\text { de dor e queimação foram maiores nesses pacientes. Não há evidências que suportem o uso } \\
\text { de nenhum dos cremes na prevenção de radiodermatite. Destaca-se que quando são dadas } \\
\text { instruções consistentes de cuidado com a pele, como lavar com sabão neutro e água, não há } \\
\text { benefício adicional sintomática adquirida pela aplicação de um creme para a área de tratamen- } \\
\text { to. Os resultados não apoiam a introdução deste tratamento preventivo caro na prática diária }\end{array}$ \\
\hline
\end{tabular}


Quadro 4 - Características dos ensaios clínicos. Curitiba, 2012

\begin{tabular}{|c|c|c|c|}
\hline Referência, ano & Sujeitos & Intervenção & Controle \\
\hline 16,2002 & $\begin{array}{l}12 \text { pacientes } \\
\text { com câncer de } \\
\text { cabeça e pes- } \\
\text { coço em trata- } \\
\text { mento radiote- } \\
\text { rápico }\end{array}$ & 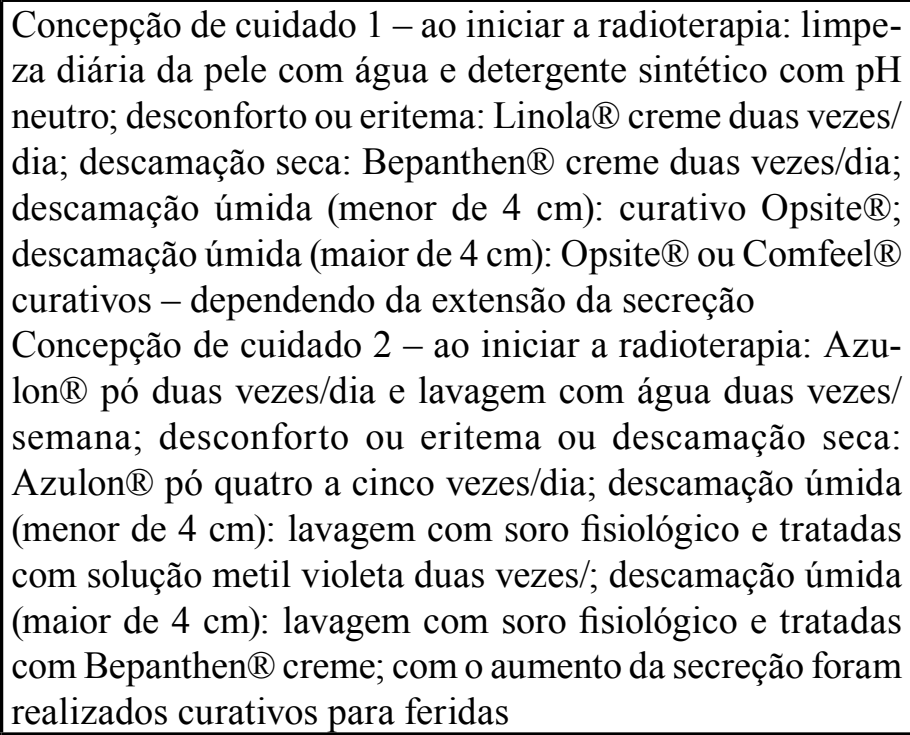 & Não se aplica \\
\hline 17,2008 & $\begin{array}{l}57 \text { pacientes } \\
\text { com câncer de } \\
\text { cabeça e pes- } \\
\text { coço, mama, } \\
\text { próstata, reto } \\
\text { ou ovário em } \\
\text { tratamento ra- } \\
\text { dioterápico } \\
\end{array}$ & $\begin{array}{l}\text { Administração de SOD } 40 \mathrm{mg} \text { pomada duas vezes/dia - } \\
\text { início em graduação de toxicidade acima de } 2 \text { e término } \\
\text { quando graduação de toxicidade } 0 \text { ou } 12 \text { semanas após o } \\
\text { início do tratamento }\end{array}$ & Não se aplica \\
\hline 18,2006 & $\begin{array}{l}506 \text { pacientes } \\
\text { com câncer de } \\
\text { cabeça e pes- } \\
\text { coço em trata- } \\
\text { mento radiote- } \\
\text { rápico }\end{array}$ & $\begin{array}{l}\text { Emulsão de Trolamine } ₫ \text { profilática: aplicação três vezes/ } \\
\text { dia desde o primeiro dia de radioterapia até duas semanas } \\
\text { após o término do tratamento } \\
\text { Emulsão de Trolamine } ₫ \text { para intervenção: aplicação ape- } \\
\text { nas após sinais de radiodermatite, três vezes/dia até duas } \\
\text { semanas após o tratamento }\end{array}$ & $\begin{array}{l}\text { Produto padrão } \\
\text { da instituição } \\
-14 \text { produtos } \\
\text { diferentes }\end{array}$ \\
\hline 19,2004 & $\begin{array}{l}357 \text { pacientes } \\
\text { com câncer de } \\
\text { cabeça e pes- } \\
\text { coço, mama ou } \\
\text { anorretal em } \\
\text { tratamento ra- } \\
\text { dioterápico }\end{array}$ & $\begin{array}{l}\text { Creme aquoso ou creme de sucralfato, ambos duas vezes/ } \\
\text { dia a partir do primeiro dia de radioterapia até duas sema- } \\
\text { nas após término do tratamento ou até resolução total da } \\
\text { radiodermatite }\end{array}$ & $\begin{array}{l}\text { Não utilizaram } \\
\text { nenhum creme }\end{array}$ \\
\hline
\end{tabular}

\section{DISCUSSÃO}

Os ensaios clínicos incluídos na presente revisão abordaram medidas para prevenção e tratamento de radiodermatite relacionada ao tratamento do câncer em diversas regiões anatômicas. Entretanto, destaca-se que nos pacientes com câncer de cabeça e pescoço é mais comum o desenvolvimento desta complicação, devido a pele mais sensível e presença de pregas, causando umidade e fricção constantes, o que a torna mais suscetível.
Ademais, são pacientes que possuem estado nutricional desfavorável, com baixo tecido adiposo na região irradiada, resultando em maior fragilidade cutânea ${ }^{(7,20)}$.

Frequentemente os pacientes com câncer de cabeça e pescoço realizam tratamento radioterápico concomitante com quimioterapia, o que potencializa o desenvolvimento da radiodermatite ${ }^{(7,20)}$. Destaca-se que esse dado corrobora com os ensaios clínicos da presente revisão que demonstram maior incidência dessa reação nos pacientes submetidos a tratamentos 
concomitantes (Evidência 1B e 1B) $)^{(18-19)}$.

Um dos fatores relacionados ao paciente que pode interferir na reação de pele e na qualidade da cicatrização é o fumo ${ }^{(21)}$. Salienta-se que um ensaio clínico aponta que os pacientes fumantes apresentaram maiores escores de toxicidade da pele, assim como os ex-fumantes (Evidência 1B) ${ }^{(20)}$. Fato dissonante dos resultados de outro estudo, que constata que não houve diferença de toxicidade entre os grupos de pacientes que nunca fumaram, ex-fumantes ou fumantes (Evidência 1B) ${ }^{(19)}$.

As orientações de cuidados necessários com a pele, durante o tratamento e dos produtos a serem utilizados na radiodermatite, dependem da graduação de toxicidade, conforme avaliação do enfermeiro e aplicabilidade de cada produto. Existem diversas recomendações de intervenções e/ou produtos, tais como aplicação de compressas com chá de camomila ou água filtrada, loção a base de ácidos graxos essenciais (AGE) ou ácidos graxos insaturados (AGI), aloe vera, sulfadiazina de prata $1 \%$, entre outros ${ }^{(6,22)}$. Entretanto, estas intervenções não possuem pesquisas clínicas que subsidiem evidências científicas que as sustente, conforme demonstrado pelos resultados da presente revisão.

Estudo de revisão de literatura sistematizada, sem metanálise, constatou que chá de camomila, AGE, aloe vera, hidrocolóide e sulfadiazina de prata $1 \%$ são utilizados em algumas instituições na prevenção e/ou tratamento de radiodermatite, os quais necessitam de estudos estruturados para avaliação de sua efetividade e constatação de evidências ${ }^{(5)}$.

Algumas pesquisas salientam a utilização de outras modalidades terapêuticas, como o uso de fitoterápico, sendo uma delas a calêndula officinalis, devido as suas propriedades antiinflamatória, antisséptica, cicatrizante, antiflogística, antialérgica, antiedematosa, calmante e refrescante ${ }^{(22-24)}$. Entretanto, há evidência que sustente sua utilização apenas para pacientes em tratamento radioterápico para o câncer de mama, conforme demonstrado por ensaio clínico que comparou seu uso com Trolamine ${ }^{\circledR}$ e obteve como resultado redução da dor local, da incidência de dermatite grave e da interrupção do tratamento no grupo tratado com calêndula ${ }^{(10)}$.

O Trolamine ${ }^{\circledR}$ consiste em uma solução de trietolamina e ácido salicílico, classificado como cicatrizante $^{(25)}$. Existem três produtos comerciais disponíveis no Brasil com o mesmo princípio ativo do Trolamine ${ }^{\circledR}$, assim como duas formas de apresentação: emulsão tópica e pomada ${ }^{(25)}$. Quando indicado para prevenção e tratamento de radiodermatite, ensaio clínico randomizado evidenciou que não há vantagem do produto na redução da incidência de radiodermatite grau dois ou maior, quando comparado a outros tratamentos (Evidência 1B) ${ }^{(18)}$.

Ensaio clínico que avaliou tratamento com Bepanthen ${ }^{\circledR}$ creme, Linola ${ }^{\circledR}$ creme, curativos Opsite ${ }^{\circledR}$ e Comfeel ${ }^{\circledR}$ e Azulon ${ }^{\circledR}$ pó não demonstrou diferença significativa com relação à prevenção de lesões (Evidência $2 \mathrm{~B})^{(16)}$. O Bepanthen ${ }^{\circledR}$ é um composto de dexpantenol, disponível comercialmente no Brasil nas apresentações pomada e solução tópica. A Linola®, composto por ácido linoléico, e Azulon ${ }^{\circledR}$ pó não são produtos registrados pela Agência Nacional de Vigilância Sanitária (ANVISA) para utilização em nosso país. Ambos os curativos citados no ensaio clínico, Opsite ${ }^{\circledR}$ e Comfeel ${ }^{\circledR}$, estão disponíveis para utilização no Brasil ${ }^{(25)}$.

A dismutase superoxidase (SOD) é uma enzima com ação antioxidante, que apresentou resultados satisfatórios quando avaliada em ensaio clínico, tanto na prevenção do agravamento da radiodermatite quanto na remissão da lesão (Evidência 2C) ${ }^{(17)}$. Entretanto, destaca-se que este produto não consta na relação de produtos liberados para uso no Brasil, segundo a ANVISA ${ }^{(25)}$.

$\mathrm{O}$ creme de sucralfato e o creme aquoso foram avaliados em ensaio clínico, não havendo evidências para utilização de um em detrimento do outro (Evidência $1 B)^{(19)}$. O sucralfato é um produto liberado para utilização no Brasil, entretanto apenas sob a forma de comprimido ou suspensão oral, e não sob a forma de creme ou pomada conforme empregado no ensaio clínico acima descrito ${ }^{(25)}$.

Destaca-se que a incorporação dos produtos citados nos ensaios clínicos que compõem a presente revisão na prática clínica dos hospitais que atendem pacientes em tratamento radioterápico para câncer de cabeça e pescoço fica dificultada. Inicialmente, pelo fato de grande parte dos produtos não terem liberação da ANVISA, mas principalmente pela falta de evidências científicas fortes que sustentem sua utilização na prevenção e no tratamento da radiodermatite.

Desta forma, deve-se preconizar outros cuidados para prevenção da radiodermatite, tais como, aumento da ingesta hídrica ( 2 a 3 litros diariamente); evitar o consumo de bebidas alcoólicas e fumo; evitar expor a área irradiada ao sol, calor e/ou frio; manter a pele no campo de tratamento seca (exceto na utilização de produtos) e livre de irritações; não utilizar qualquer produto no local antes das seções 
de radioterapia; evitar coçar, arranhar, esfregar ou escovar o local de tratamento; lavar a pele do campo de tratamento com água morna, evitando banhos muito demorados e com a água muito quente ou muito fria; utilizar produtos de higiene com $\mathrm{pH}$ neutro ou infantil; entre outros ${ }^{(6,23)}$. Estudo clínico ressalta que um aconselhamento consistente, a higiene da pele e uma avaliação regular realizada pelo profissional pode ser extremamente eficaz na minimização da radiodermatite (Evidência 1B) ${ }^{(20)}$.

Salienta-se que são incipientes os estudos baseados em evidências científicas que indiquem a realização de cuidados de enfermagem ou a utilização de produtos na prevenção e/ou tratamento de radiodermatite, efeito adverso que pode ser bastante doloroso e desconfortável ao paciente ${ }^{(8)}$. Assim, enfatiza-se a relevância de ensaios clínicos na área de Enfermagem Oncológica, que são essenciais para implementação de um cuidado seguro, eficiente e de qualidade ao indivíduo.

\section{CONSIDERAÇÕES FINAIS}

Infere-se que a maioria dos estudos não demonstra evidências para introdução de um produto/cuidado para prevenção e/ou tratamento de radiodermatite, não tendo, desta forma, apoio na prática profissional diária. Ademais, na presente revisão não houve predominância na utilização de nenhum produto para prevenção e/ou tratamento dessa reação. $O$ único produto que teve sua eficácia comprovada foi o SOD, porém este estudo não se desenvolveu em comparações com outros produtos e seu nível de Evidência é 2C. Além disso, este produto não está disponível no Brasil.

Apesar de a radiodermatite configurar uma reação com incidência elevada e graves complicações ao paciente, destaca-se a escassez de estudos sobre o tema, conforme evidenciado na presente revisão. Faz-se necessário direcionar as pesquisas para esta temática, a fim de incorporar tecnologias ou produzir inovações que beneficiem os pacientes suscetíveis ou acometidos por esta complicação. Indica-se a realização de ensaios clínicos cegos e multicêntricos, a fim de produzir evidências científicas fortes que sustentem a prática clínica.

Enfatize-se que a radiodermatite é um efeito adverso que pode ser prevenido, ou minimizado, por meio de orientações e intervenções prévias aos pacientes, familiares e/ou acompanhantes/cuidadores sobre os cuidados com a pele, uma vez que esses realizam os cuidados também em domicílio, contribuindo com a integridade cutânea do local do tratamento. Os enfermeiros interagem de forma direta com os pacientes, e cabe a esses profissionais oferecer informações completas e específicas. Destaca-se que a enfermagem é importante desde o início do tratamento até a sua conclusão, sendo a prevenção de complicações e a qualidade de vida os focos maiores de cuidado.

\section{REFERÊNCIAS}

1. Ministério da Saúde (BR). Secretaria de Atenção à Saúde. Instituto Nacional de Câncer. TNM: classificação de tumores malignos. $6^{\mathrm{a}}$ ed. Rio de Janeiro: INCA; 2004.

2. Simões JC, Gama RR, Winheski MR. Câncer: estadiamento e tratamento. São Paulo: Lemar; 2008.

3. Instituto Nacional de Câncer José Alencar Gomes da Silva. Estimativa 2012: incidência de câncer no Brasil. Rio de Janeiro: INCA; 2011. [acesso em 10 jul 2012]. Disponível: http://www.inca.gov.br

4. Fisher J, Scott C, Scarantino CW, Leveque FG, White RL, Rotman M, et al. Phase III quality of life study results: impact on patients quality of life to reducing xerostomia after radiotherapy for head and neck cancer - RTOG 97-09. Int J Radiat Oncol Biol Phys. 2003;56(3):832-6.

5. Blecha FP, Guedes MTS. Tratamento de radiodermatite no cliente oncológico: subsídios para intervenções de enfermagem. Rev. bras. cancerol. 2006;52(2):151-63.

6. Denardi UA, Matsubara MGS, Bicudo FG, Okane ES, Martins CA, Moscatello E. Enfermagem em radioterapia. $1^{\text {a }}$ ed. São Paulo: Lemar; 2008.

7. Instituto Nacional de Câncer. Ações de enfermagem para o controle do câncer: bases do tratamento do câncer. $2^{\mathrm{a}}$ ed. Rio de Janeiro: INCA; 2002. [acesso em 10 out 2010]. Disponível: http://bvsms.saude.gov.br/bvs/ publicacoes/inca/acoes_cap6.pdf.

8. Dealey C. Cuidando de feridas: um guia para as enfermeiras. $3^{\mathrm{a}}$ ed. São Paulo: Atheneu; 2008.

9. Candido LC. Nova abordagem no tratamento de feridas. São Paulo: SENAC-SP; 2001.

10. Prommier P, Gomez F, Sunyach MP, D'Hombres A, Carrie C, Montbarbon X. Phase III randomized trial of Calendula officinalis compared with Trolamine for the prevention of acut dermatitis during irradiation for breast cancer. J Clin Oncol. 2004;22(8):1447-53. 
11. De Conno F, Ventafridda V, Saita L. Skin problems in advanced and terminal cancer patients. J Pain Symptom Manage. 1991;6(4):2547-60.

12. Mendes KDS, Silveira RCCP, Galvão CM. Revisão integrativa: método de pesquisa para a incorporação de evidências na saúde e na enfermagem. Texto Contexto Enferm. 2008;17(4):758-64.

13. Glasziou P, Del Mar C, Salisbury J. Prática clínica baseada em evidências: livro de exercícios. $2^{\mathrm{a}}$ ed. Porto Alegre: Artmed; 2010.

14. Nobre MR, Bernardo WM, Jatene FB. A prática clínica baseada em evidencias. Parte I - Questões clínicas bem construídas. Rev. Assoc. Med. Bras. 2003;49(4):445-9.

15. Ministério da Saúde (BR). Diretrizes metodológicas para elaboração de pareceres técnico-científicos para o Ministério da Saúde. Brasília: Ministério da Saúde; 2007 [Internet] [acesso em 19 jul 2011]. Disponível: http://iah.iec.pa.gov.br/iah/fulltext/glossario/diretrizes_ metodologicas_ptc_conteudo_ago_2007.pdf

16. Schreck U, Paulsen F, Bamberg M, Budach W. Intraindividual comparison of two different skin care conceptions in patients undergoing radiotherapy of the head-and-neck region. Creme or powder? Strahlenther Onkol. 2002;178(6):321-9.

17. García ÁM, Carrizosa MCL, Ocaña CV, Ots PS, Pérez JMD, Accame EC, et al. Superoxidase dismutase (SOD) topical use in oncologic patients: treatment of acute cutaneous toxicity secondary to radiotherapy. Clin Transl Oncol. 2008;10(3):163-7.

18. ElliottEA, Wright JR, Swann S, Nguyen- Tân F, Takita C, Bucci MK, et al. Phase III trial of an emulsion containing trolamine for the prevention of radiation dermatitis in patients with advanced squamous cell carcinoma of the head and neck: results of radiation therapy oncology group trial 99-13. J Clin Oncol. 2006;24(13):2092-7.

19. WellsM,MacmillanM,RaabG,MacBrideS,BellN,MacKinnon $\mathrm{K}$, et al. Does aqueous or sucralfate cream affect the severity of erythematous radiation skin reactions? A randomised controlled trial. Radiother Oncol. 2004;73(2):153-62.

20. Salvajole JV, Souhami L, Faria SL. Radioterapia em oncologia. $1^{\text {a }}$ ed. Rio de Janeiro: Medsi; 1999.

21. Porock D, Kristjanson L, Nikoletti S, Cameron F, Pedler P. Predicting the severity of radiation skin reactions in women with breast cancer. Oncol Nurs Forum. 1998;25(6):1019-29.
22. Matsubara MGS, Villela DL, Hashimoto SY, Reis HCD, Saconato RA, Denardi UA, et al. Feridas e estomas em oncologia: uma abordagem interdisciplinar. São Paulo: Lemar; 2012.

23. Sartori LR, Ferreira MS, Perazzo FF, Mandalho LL, Carvalho JCT. Atividade antiinflamatória do granulado de Calendula officinalis L e Matricaria recutita. Rev Bras Farmacogn. 2003;13(1):17-9.

24. Carmo LHA. Avaliação em cultura celular da atividade farmacológica e desenvolvimento de formulações contendo extrato padronizado de Calendula officinalis L. [tese]. São Paulo (SP): Faculdade de Ciências Farmacêuticas - Universidade de São Paulo; 2003.

25. Agência Nacional de Vigilância Sanitária(BR). Consulta de produtos e medicamentos. Brasília: ANVISA [Internet] 2012. [acesso em 23 jul 2012]. Disponível: http://www7.anvisa.gov.br/datavisa/consulta_produto/ Medicamentos/frmConsultaMedicamentos.asp. 